\title{
The Road to Sustainable Exercise: Using Stationary Bicycles to Power a Green Gym
}

\author{
Basel Hindi, Nasser Atiyah, Sami Abdalla, Omar Al Ani, Shameel Abdulla, Reza Tafreshi \\ Texas A\&M University at Qatar, Qatar Foundation, \\ Doha, Qatar \\ basel.hindi@qatar.tamu.edu; nasser.atiyah@qatar.tamu.edu; sami.abdalla@qatar.tamu.edu
}

\begin{abstract}
The purpose of this study is to determine the amount of electric power that a stationary bike can produce. The end goal is to determine if it is feasible to power an entire "green gym" using only stationary bikes. This study is significant because the process of configuring a stationary bike to produce electricity is simple, and therefore, could potentially be implemented in gyms worldwide. Non renewable energy sources are quickly depleting, and the search for alternative energy is at its height, which is precisely why this study is of importance. The energy generated by pedalling the bike was captured and converted into electrical energy using a DC generator. Power input and electric power output were measured using a power meter and an electrical load circuit along with a data acquisition system respectively. The efficiency was determined to be $(51 \pm 4.3) \%$ within a confidence interval of $95 \%$. Five male subjects of age $19.5 \pm 0.5$ years participated in the study. Each produced 54 watts on average over the course of a 10 minute workout. Considering a small gym would require approximately 265 watts, it would require five bikes to continuously power the gym. This result proves that pedal power can be utilized as an alternative energy source for low power consumption applications.
\end{abstract}

Keywords: Power Efficiency Stationary Bicycle Energy Conservation Green Gym

\section{Introduction}

Energy conservation has always been of the utmost importance to human civilization. Since most of the world's energy is generated by non-renewable fossil fuels, it is inevitable that these will run out. Some countries have made great steps towards using renewable energy. For instance, Norway derives more than $80 \%$ of its power from renewable energy sources [1]. While there are still many obstacles to overcome before the world becomes solely dependent on renewable energy, possible solutions can be tested for feasibility.

The purpose of this paper is to determine the amount of electric power that a stationary bike can produce and if it would be enough to power a hypothetical "green gym". A power meter will measure values for power input (power at the pedal), and an electrical load circuit will be used to convert the rotational kinetic energy of the bike wheel into electric power. The power meter has a built in data acquisition system, which sends the acquired data to a mobile phone application, where the data is logged and compiled. The DC motor's power output can be logged using LabVIEW. The compiled information is then exported and analyzed. The power output can then be aligned and compared to the power input to find efficiency, which will indicate the feasibility of using bikes as a source of electrical power.

A stationary bike was modified to incorporate a power meter and a DC motor in its setup. Power measured at the pedal requires values of angular velocity and torque. This was done through the use of a bicycle power meter that measures the angular velocity and torque at a significantly higher accuracy than regular strain gauges. [2][3] Bicycles in general, can have mechanical efficiencies between 80 to $99 \%$ which gave merit to the idea that powering a gym through the use of stationary bicycles may be feasible. Various studies have shown that the average human inputs between $120 \mathrm{~W}$ to $200 \mathrm{~W}$. [4][5] Lastly, mechanical energy can be converted into electrical energy at efficiencies ranging between $35 \%$ to $60 \%$ depending on the setup. This provided valuable insight into the possible power that could be generated after losses within the bike itself, and throughout energy conversion from mechanical to electrical [6][7][8].

The following power consumption values provide some insight into the type of gym that could be powered by stationary bikes alone. LED light bulbs consume 4-6 watts each, DC powered portable fans consume 25 - 55 watts, and small DC powered LCD TVs consume approximately 12 watts. [9] Thus, assuming a "green gym" contains three fans, 20 
light bulbs, and five small LCD screens, approximately 265 watts would need to be supplied to the gym from the stationary bikes.

Furthermore, other experimental approaches use different apparatus to convert the rotational kinetic energy of the bike wheel into electrical energy. One such approach is to convert the energy using electromagnetic energy harvesting. This is done by converting the rotational motion into one dimensional motion (like a magnet moving up and down). An electric conductor is rotated in the presence of a magnetic field. This induces a voltage difference across the conductor. The induced voltage can be used to drive electric loads. The electromagnetic approach generates AC power, which is then converted into DC power to charge a battery. [10] The approach discussed in this paper generates DC power without the need for any conversions from AC by replacing the electromagnetic apparatus with a DC motor and other equipment, as discussed next.

\section{Experimental Set Up}

This section discusses the experimental set up including the apparatus, software, and experimental procedure.

\subsection{Apparatus}

The experimental set up consisted of the following hardware:

1) Stages- Ultegra Power Meter: The power meter (1) is a sensor that findsthe input power from the user's left foot onto the pedal of the bike. It assumes the user has symmetrical power input from both legs, and thus, multiplies all its measured values by two. It has a built-in data acquisition system, which can be paired with a data logging phone application through Bluetooth. The data collected can then be uploaded to the internet and accessed by a computer.

2) DC Motor: A 250 watt DC motor (2) was utilized as a generator and voltage source throughout this study. It converts rotational kinetic energy into electric power. This component was attached to the moving wheel of the stationary bike.

3) DC Motor Attachment Gear: The DC motor was attached to the stationary bike wheel directly. DC motors have a range of revolutions per minute at which they function. In order to prevent the DC motor from exceeding this range (maximum RPM of 2500), a gearing ratio between the stationary bike wheel and the attachment gear was calculated to be 6:1. Thus, a DC motor shaft attachment (3) was machined to make this gearing ratio possible. 


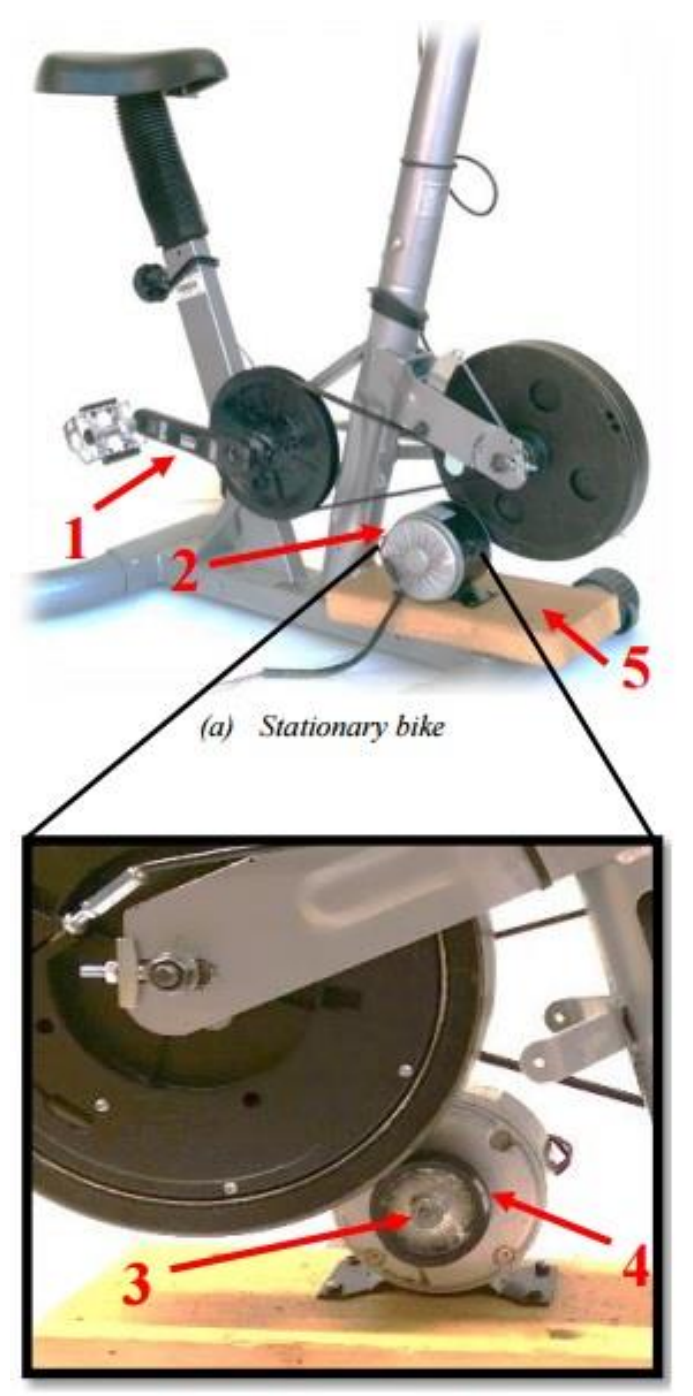

(b) DC Motor and fixture set up

Fig. 1: Experimental Set Up (1) Power meter, (2) DC Motor, (3) Attachment gear, (4)Rubber Traction Sleeve, (5) DC Motor Fixture.

4) Rubber Traction Sleeve: A rubber traction sleeve (4) was press-fitted onto the DC motor attachment to increase the coefficient of friction and eliminate slip between the DC motor attachment and the wheel of the stationary bike. The rubber also needed to have heat-withstanding properties due to the high friction and heat it experiences.

5) DC Motor Fixture: In order for the DC motor to stay snugly attached to the stationary bike wheel (and thus reduce slip), a block of wood (5) was attached to both the frame of the bike and the DC motor.

6) NI Elvis: The NI Elvis was utilized to acquire data from the DC motor. A circuit comprised of one 7.5 ohm resistor, one $0.035 \mathrm{ohm}$ resistor, and a DC motor was connected to the NI Elvis.

\subsection{Software}

The experimental set up required use of the following software:

A Training Tracker: A Training Tracker is used by professional cyclists to track their progress against different variables over time. This software was the data logging application used to send the data collected by the power meter to a computer.

LabVIEW by National Instruments: A LabVIEW virtual instrument was developed and utilized to determine power output given the value of a known resistor and the values of voltage acquired by the DAQ. LabVIEW then logged the power data. 


\subsection{Experimental Procedure}

To find efficiency, both power input and power output was measured. In order to measure power input, a power meter (Part No. 1, Figure 1a) was attached to the stationary bike. The power meter transmitted the data via Bluetooth, which required use of a free mobile phone application for data logging called "A training tracker".

In terms of the setup for the DC motor, a cylindrical piece (Part No. 3, Figure 1b) was machined onto the motor and connected to the stationary bike's wheel (further discussed in Section 4). In order to design the attachment gear, the subjects were asked to pedal at maximum capacity, and the angular velocity of the stationary bike wheel was measured using a tachometer. The gearing ratio for the attachment gear was decided by dividing the maximum allowable rotations per minute of the DC motor (2500 RPM) by the maximum rotations per minute of the stationary bike (410 RPM). This gearing ratio was found to be $6: 1$ (from stationary bike wheel to the DC motor attachment gear). The DC motor was then fixed onto an elevated surface which ensures contact with the bike at all times.

Five subjects pedalled on the bike for 10 minutes each at their preferred pace and their runs were recorded. Ten minutes was selected as the run time because it is a good representation of the average workout on a stationary bike. A mobile phone application was used to acquire the power input data. These values were then uploaded to Microsoft Excel. Additionally, the power generated from the rotation of the DC motor was determined through use of a circuit connected to the DAQ, which acquires data for voltage. The DC motor was used as the voltage source and a virtual instrument was used to determine power through voltage and resistance. The details of the power output calculation are discussed in the next section.

\section{Power Output and Efficiency Calculation}

A circuit consisting of the DC motor, a $7.5 \Omega$ resistor and a $0.035 \Omega$ resistor was built. The $7.5 \Omega$ resistor, which was simply a cartridge heater used in 3D printer nozzles, was used as a load resistor due to the high current that a heating element can withstand. The fact that this resistor was $7.5 \Omega$ is also important, because it provided high output power values without putting huge amounts of strain on the subjects. Lastly, using a load resistor is important because it acts as a load, causing current to flow in the circuit. While this made pedalling more strenuous, it also increased the power output values. The $0.035 \Omega$ resistor was used as a sensing resistor and voltage was measured across it. Figure 2 displays this circuit.

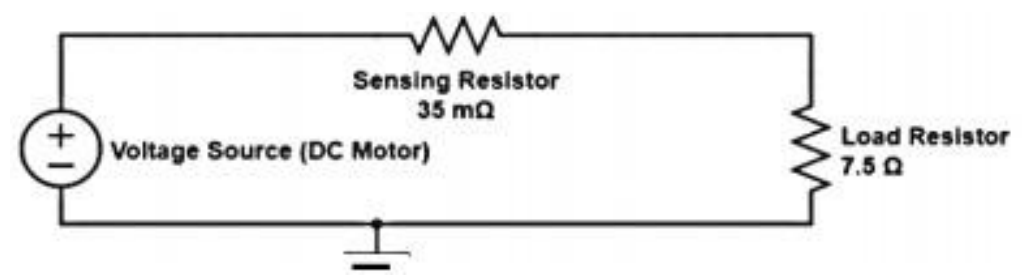

Fig. 2: NI Elvis Circuit.

After acquiring voltage across the sensing resistor, power output can be determined by:

$$
P=V^{2} / R
$$

Then the power input and power output data was filtered, and the efficiency was calculated and statistically analysed using a normal distribution test. The filtering method utilized was to take the average of every five readings (refer to Section 4). This method was selected because a tiny misalignment in the power output and input readings could cause huge, unrealistic spikes in efficiency. Thus, the averaged efficiency can be determined by:

$$
\text { Efficiency }=\frac{\sum_{i=1}^{5}{\text { DC Motor } \text { Power }_{i}}^{5}}{\sum_{i=1}^{5} \text { Pedal Power }_{i}}
$$

\section{Results}


As discussed earlier, five subjects cycled for approximately ten minutes each. The power meter acquired all necessary data for power input, and sent it to a data-logging software at a sampling rate of $1 \mathrm{~Hz}$. The power output was measured using a circuit connected to the DC motor. The resistance and voltage were measured, which provided values for power at a frequency of $1 \mathrm{~Hz}$. This sampling rate could have been changed on LabVIEW, but was selected to match the sampling rate of the power meter.

Figure 3a shows the aligned power input (upper lines) and output (lower lines). Figure 3b shows the efficiency of Subject 3. The power output is the approximate translation of power input (with lower magnitude). At some points, power output and input became slightly misaligned, yielding unrealistic efficiencies. These misalignments were likely a result of the differences in sensory equipment. The power meter measuring power input is highly accurate with constant calibration and fast dynamic response. The DC motor (being used to measure power output) has a slow dynamic response. [11] To combat this, filtering the data by averaging was necessary (Equation 1). The only remaining variable was to find out how many data values should be averaged in order to filter out the misalignments, but retain the significance and meaning of the data. Figure 3 also shows three viable options that were available when analysing the data collected. The interval with which the data was averaged was examined for 3 seconds, 5 seconds, and 10 seconds.

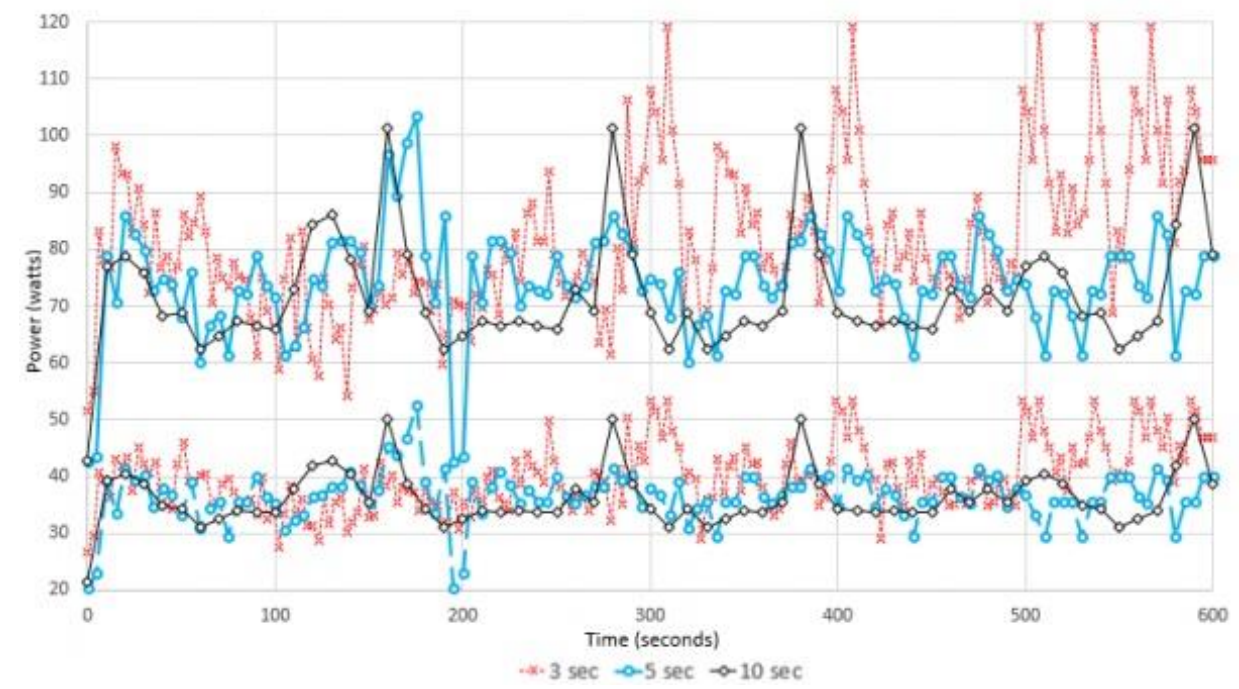

Fig. 3a: Subject 3's input and output when averaging the results every 3, 5 and 10 seconds.

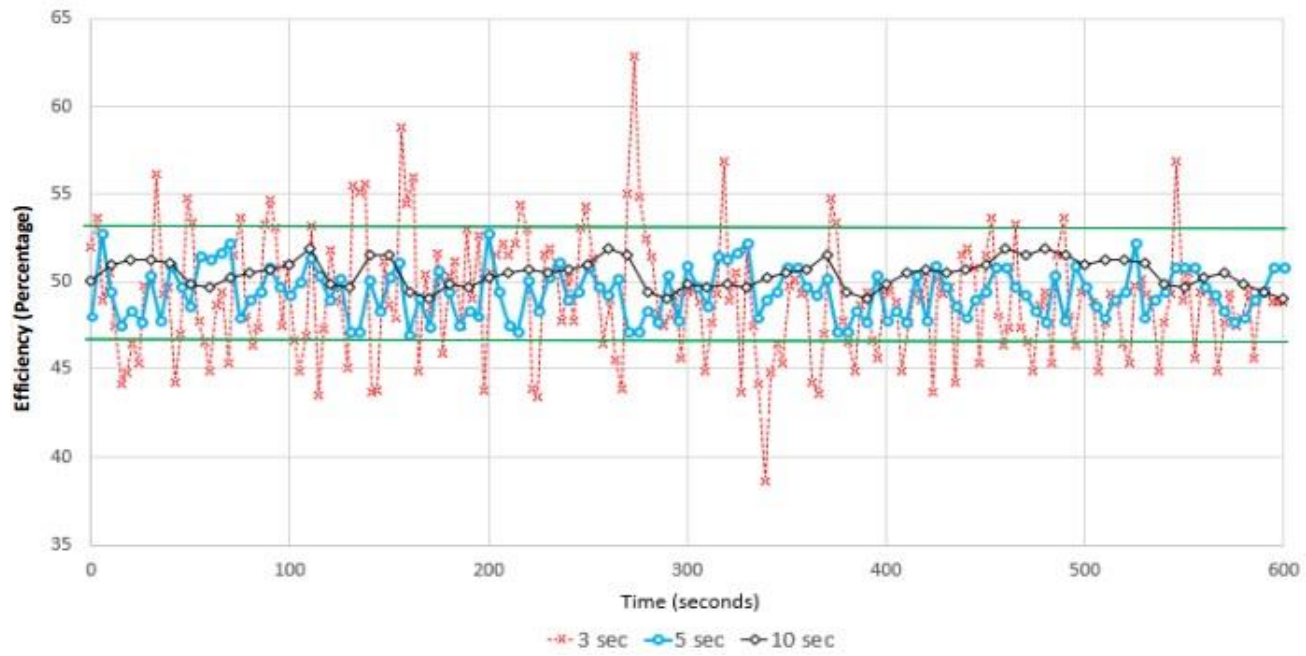

Fig. 3b: Subject 3's efficiency when averaging the results every 3,5 and 10 seconds.

Table 1 represents the statistical difference between the three averaging intervals. 
Table 1: Mean and standard deviation for Subject 3 at each of the time intervals.

\begin{tabular}{|c|c|c|c|}
\hline Interval (s) & Mean $(\boldsymbol{\mu})(\mathbf{W})$ & Standard Deviation $\left(\boldsymbol{\sigma}_{\mathbf{x}}\right) \mathbf{( W )}$ & Range at Confidence level of 95\% (1.96 $\boldsymbol{\sigma})(\mathbf{W})$ \\
\hline 3 & 49.038 & \pm 3.448 & \pm 6.758 \\
\hline 5 & 49.385 & \pm 1.457 & \pm 2.856 \\
\hline 10 & 49.569 & \pm 0.814 & \pm 1.595 \\
\hline
\end{tabular}

According to the findings in Table 1 and Figures $3 \mathrm{a}$ and 3b, it was decided that averaging every 5 seconds of data and taking this value as one data point, would be done for each of the five test runs. This 5 second averaging method was chosen over two other averaging options, which were averaging every 3 and 10 seconds. The reason the 3-second averaging method wasn't selected was simply because the standard deviation it presented was too high, indicating that misalignments within the data were problematic. On the other hand, the reason that the 10 -second averaging method wasn't used was because although it has a rather low standard deviation and confidence level range, it averages too many of the data points, making the results less meaningful. The 5-second averaging method has an acceptable standard deviation while still properly representing the results. The green lines shown in Figure $3 b$ show a graphic representation of how the 5 second averaging method is able to best approximate the calculated results. Figure 4 shows the efficiency values for each of the test subjects plotted onto one graph. In order to decrease the errors in the efficiency, the 5-second averaging method mentioned above was used. The uncertainty bars illustrate the standard deviation and mean for each of the five subjects.

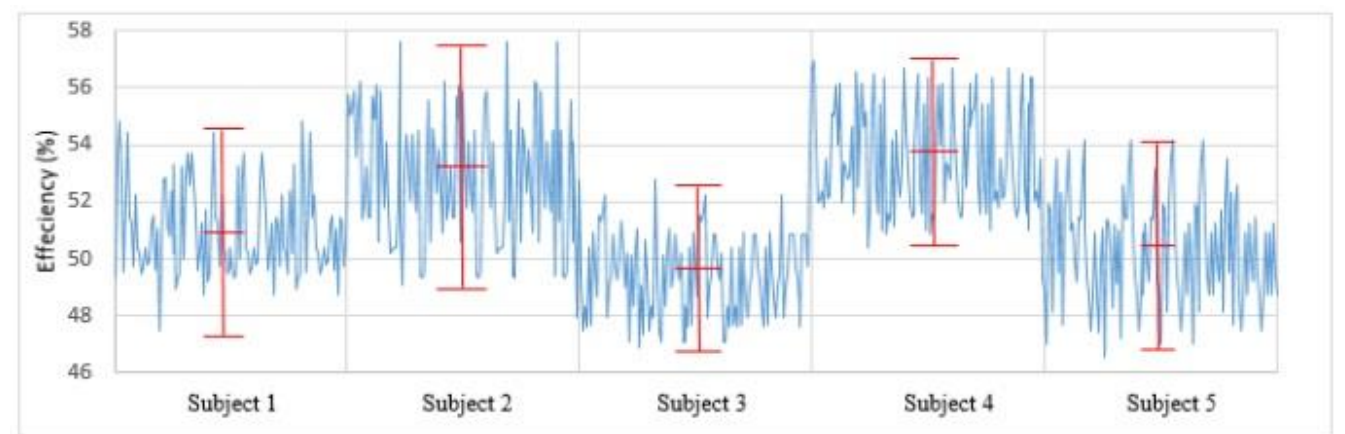

Fig. 4: The efficiencies for all 5 subjects at 5 second averaging. The red uncertainty bars illustrate the mean (half way mark) along with a $95 \%$ confidence interval for each of the subjects' trials.

The average efficiency of the stationary bike for all test subjects was calculated to be $(51 \pm 4.3) \%$ within a confidence interval of $95 \%$.

\section{Discussion}

If the "green gym" consists of three fans, 20 light bulbs, and five small LCD screens (one per bike), it would require five stationary bikes producing 54 watts each to power the gym.

The low uncertainty in the average efficiency $(51 \pm 4.3) \%$ indicates that efficiency stayed relatively constant throughout every trial. Energy losses varied slightly at different inputs, causing slight changes in efficiency. If the test subjects were to pedal at a consistent rate, while maintaining the same power input, the average efficiency would be much more accurate than what it currently is. However, since the test subjects naturally fluctuated in their power input throughout their workout, the energy losses due to the system fluctuated accordingly.

Bicycles in general, are known to be the most efficient form of transportation with a mechanical efficiency ranging between $80 \%$ and $99 \%$. The bike used in this experimental set up had an average efficiency of $(51 \pm 4.3) \%$. Friction and heat within the gears and belt of the bike impacted the results, and can be reduced to help improve the overall efficiency of the set up. Lubricating the gears and fitting a tighter belt (to prevent slip) can help accomplish this goal. The conversion of 
mechanical energy to electrical energy through the DC motor can also be made more efficient by better optimizing the size of the DC motor to the power output being generated by the bike.

After each of the five subjects pedalled for 10 minutes, their power outputs were averaged, and it was found to be approximately 54 watts. The average power input was found to be approximately 105 watts. As discussed earlier, the average human being can produce 120-200 watts on a standard bicycle. The discrepancy may be a result of the load resistor selected. As previously discussed, altering the load resistor changes how strenuous the workout is, and thus, impacts power input. Altering the resistor would make pedalling more difficult, but would result in higher current flowing in the circuit, and thus, higher power outputs. To maximize power, a larger load resistor could be implemented.

Furthermore, the apparatus can be applied to gyms worldwide, especially considering the simplicity of the set up, and the relatively low cost of the equipment. The following table displays the approximate costs of converting a stationary bike into an electric generator.

Table 2: Costs of converting a stationary bike into a generator.

\begin{tabular}{|l|l|}
\hline Item & Cost (USS) \\
\hline DC Motor & 25 \\
\hline Half a meter of rubber (for rubber traction sleeve) & 7 \\
\hline Gear Attachment Material Cost & 1 \\
\hline 20 minutes of machine shop labor for gear attachment & 20 \\
\hline Total & 53 \\
\hline
\end{tabular}

In a 24 hour gym, the assumption can be made that a stationary bike will be used for 8 hours per day. The average price of $1 \mathrm{kWh}$ worldwide ranges from $\$ 0.08$ to $\$ 0.41$, however the majority of countries tend to fluctuate around the price of \$0.30. [12][13] If the average power output of 54 watts is maintained throughout the 8 hours of workout, $1.6 \mathrm{MJ}(0.44$ $\mathrm{kWh})$ are generated. Which means, every day $\$ 0.13$ could be saved by one stationary bike. At that rate, it would take approximately 410 days to begin profiting from the conversion. For most gyms, the decision to convert bikes into generators would be profitable over time. In one year (after breaking even), each bike would save approximately $\$ 47$, but more importantly, the gym could market itself as a "green gym" with self-sufficiency in terms of energy consumption.

\section{Conclusion}

This experimental approach can be altered and tweaked to serve a greater purpose in the future. Firstly, a variable resistor can be implemented in the circuit to alter the difficulty of pedalling. An easy, low power output workout can be attained by altering the resistance, and a high power output, strenuous workout can also be achieved. Secondly, the apparatus could be permanently attached to a stationary bike and sold as a complete kit to gyms. This would eliminate the process of converting the bike, and would reduce costs because parts could be machined in bulk. Lastly, the experimental set up could be expanded to charge a battery. While this would decrease efficiency, it would allow the power to be stored and used when needed, without the need for constant input from the user.

\section{References}

[1] D. Gonzalez, A. Killinc, N. Weidmann, "Renewable Energy Development Hydropower in Norway," in Hydropower Norway Seminar Paper, 2011, [Online]. Available: https://www.thnuernberg.de/fileadmin/Fachbereiche/bw/studienschwerpunkte/international_business/Master/CAIF D/SeminarPapers/HydropowerNorway_SeminarPaper.pdf

[2] P. Bakane, K. S. Zakiuddin, "Analysis of Bicycle Ergometer: A Review," International Journal of Emerging Technology and Advanced Engineering, 2013, [Online]. Available: http://www.ijetae.com/files/Volume3Issue5/IJETAE_0513_130.pdf

[3] F. Yildiz, E. P. Tau, "Potential Ambient energy-harvesting sources and techniques," Digital Library and Archives of the Virginia Tech University Libraries, 2003, [Online]. Available: https://scholar.lib.vt.edu/ejournals/JOTS/v35/v35n1/yildiz.html 
[4] N. Hibi, H. Fujinaga, K. Ishii, "Work and power outputs determined from pedalling and flywheel friction forces during brief maximal exertion on a cycle ergometer," European Journal of Applied Physiology, vol. 74, no. 5, pp. 435-442, 1996.

[5] D. D. Wilson, "HUMAN POWER," in International Human Powered Vehicle Association, 2011, [Online]. Available: http://www.ihpva.org/HParchive/PDF/hp52-2001.pdf

[6] Ş. Mocanu, A. Ungureanu, R. Varbanescu, "Bike-Powered Electricity Generator," in Asia Pacific Journal of Multidisciplinary Research, 2015, [Online]. Available: http://www.apjmr.com/wpcontent/uploads/2015/02/APJMR-2015-3-172-Bike-Powered-ElectricityGenerator.pdf

[7] B. Pelz, J. Feiereisen, "Bicycle Powered Generator for the University Farm," in Washington University, St. Louis Electrical And Systems Engineering., [Online]. 2010, Available: http://ese.wustl.edu/ContentFiles/Research/UndergraduateResearch/CompletedProjects/WebPages/sp 10/FeiereisenPelz/Feiereisen\%20and\%20Pelz.pdf

[8] R. Suhalka, M. Khandelwal, K. Sharma, A. Sanghi, "Generation of Electrical Power using Bicycle Pedal," International Journal of Recent Research and Review, 2014, [Online]. Available: http://www.ijrrr.com/papers72/10- Generation\%20of\%20Electrical\%20Power\%20using\%20Bicycle\%20Pedal.pdf

[9] R. Megalingam, P. Veliyara, R. Prahbu, R. Katoch, "Pedal Power Generation," International Journal of Applied Engineering Research, 2012, [Online]. Available: http://gimt.edu.in/clientFiles/FILE_REPO/2012/NOV/23/1353644745242/60.pdf

[10] S. David, "Harvesting Electrical Energy from a Stationary Bike: An Experimental Approach," in Massey University, 2015, [Online]. Available: http://mro.massey.ac.nz/bitstream/handle/10179/5159/02_whole.pdf

[11] M. Khan, A. Khan, U. Ghazali, "Speed Control of DC Motor under Varying Load Using PID Controller," CSC Journals, 2015, [Online]. Available: http://www.cscjournals.org/manuscript/Journals/IJE/Volume9/Issue3/IJE485.pdf

[12] "Electricity - U.S. Energy Information Administration (EIA)", Eia.gov, 2016, [Online]. Available: http://www.eia.gov/electricity/

[13] "Electricity", Iea.org, 2016, [Online]. Available: http://www.iea.org/topics/electricity/ 